\title{
SLL attenuation-based thinned antenna design for next-generation communications
}

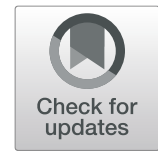

Gebrehiwet Gebrekrstos Lema* (D, Dawit Hadush Hailu and Tekle Brhane Wuneh

\begin{abstract}
Among the hot issues of the Fifth Generation (5G), developing by the Third Generation Partnership Project (3GPP), advanced antenna design significantly improves the wireless communication qualities. However, limited number of researches have been published on how the smarter antenna design satisfies the $5 \mathrm{G}$ antenna design requirements. Hence, in this paper, we have shown that effective antenna designing techniques achieve substantial antenna performance enhancements that are essential input for the current and next-generation communication engineering. First, the excitation weights of the individual array elements are optimized to obtain enhanced antenna performances including reduced side lobe level (SLL), high directivity, reduced power consumption, and flexible radiation pattern. Then, a novel SLL attenuation technique is introduced to further reduce the SLL, power wastage, and interference. In general, the proposed technique of radiation pattern synthesis has resulted in to much better SLL reduction, directivity improvement, power wastage and interference reduction.
\end{abstract}

Keywords: Antenna design, Beam forming, Antenna array, Side lobe, Directivity

\section{Introduction}

The interest in the massive wireless communication has grown quickly in recent years [1]. The 5G technology suggests that further researches are needed on how to solve the significant traffic demand due to the increasing number of users, channel allocation, and reliability issues. Among the smart antenna applications, to enhance the signal to interference ratio (SIR), the array pattern should have several nulls in the azimuth plane. One possible solution is to use a circular array pattern; however, it does not have any nulls in the azimuth plane [2]. With elliptical arrays instead of circular arrays [3], reduction of the distance of the arrays decreases the side lobes; however, the mutual coupling influence becomes another challenge. Using concentric arrays [4], uniform circular array [5], and conical arrays [6, 7] has shown better radiation characteristics. The properties of linear and circular array combination are discussed in [8]. In all of these researches, simultaneous side lobe level (SLL) reduction and directivity enhancement were challenging engineering designs. Next-generation antenna

\footnotetext{
* Correspondence: g.jcool.com@gmail.com

School of Electrical and Computer Engineering, Mekelle University, Mekelle, Ethiopia
}

design suggests even better antenna radiation characteristics including reduced antenna size, increased radiation characteristics, power efficiency, reduced antenna weight, and cheaper system costs.

Different antenna designing techniques are proposed in many antenna array synthesis problems as a solution for the 5G ambitious objectives. The concept of antenna smarting starts when the desired signal maximization and interference minimization [9-11] are achieved at a small system expense. Recently, beamforming is employed on linear antenna arrays in which the SLL was reduced meaningfully [12]. Many optimization techniques were applied to design an antenna in different complexities [13-16]. Particle swarm optimization (PSO) has used to design an elliptical-cylindrical antenna array (EcAA). However, neither the beamwidth enhancement nor the SLL reduction are sufficient to satisfy the 5G stingy requirements. Another evolutionary technique, called cat swarm optimization (CSO), has been applied on linear antenna array [18]; however, neither the side lobe reduction nor nulling of the interferer direction has shown a substantial antenna performance enhancement. Recent researches have shown that new differential evolution (DE) type of optimization techniques do have 
better and robust antenna design capability [19]. Though this optimization technique demonstrates accurate solutions than the ordinary deterministic forms of antenna array design, the question of setting their control parameters was tedious. This indicates that we really need a simple, robust, and quick designing technique.

Quite recently (2017), synthesis of a non-uniformly spaced linear antenna array [20] has been realized. Though an inter-element coupling problem is solved, the optimization technique complexity and the ability to scan the azimuth direction and further SLL reductions are still important. In [21], a novel antenna for radar applications has been studied using non-uniform excitation weights of the individual elements of the antenna array. However, since the excitation weights are not uniform the implementation is complex for practical considerations. Besides, the level of antenna performance enhancement does not guarantee the $5 \mathrm{G}$ requirements, and further antenna performance enhancement is vital. A genetic algorithm based SLL reduction was proposed [22], however, neither significant SLL reduction nor azimuthal scanning was possible with the circular antenna array. For effective antenna design, a self-adaptive DE algorithm has shown the potential of simplicity and robustness. Thinned array design based on multi-objective cross entropy algorithm is also implemented to reduce the SLL [23]. Nevertheless, the results do not reflect significant SLL reduction and the numbers of antenna array elements are much more which increases the antenna cost and weight. Recently [24], a pattern synthesis of linear antenna arrays is performed using an enhanced flower pollination algorithm, but the amount of SLL reduction is still on the range of $20 \mathrm{~dB}$ which is insufficient for the rapidly increasing number of network nodes like 5G. Further filtering the SLL enhances the power efficiency and inference reduction [25, 26]. However, the significance of the SLL attenuation on non-array antenna lacks the pattern flexibility and directivity. Flexibility of the patterns is also difficult with linear antenna array. Hence, for flexible radiation pattern synthesis, a hyper beamforming and SLL attenuation techniques are studied in this paper. In [28, 29], the effects the fractal geometrical shape and self-affine fractal structure on the entropy are evaluated, respectively. They have shown that the antenna size reduction is possible on the antenna design. However, antenna arrays are better in radiation characteristic flexibility than fractal antennas. In 2019, a feasibility study on antenna design was evaluated [30]; however, it doesn't consider any SLL attenuation technique and hence it was unable to exploit the further interference reduction, power efficiency, and thinning concepts of antenna design.

\section{Methods}

As the radiation pattern of a single-element antenna is relatively less directive, antenna arrays have shown great importance both to enhance the directivity and to reduce the SLL. Besides, hybrid antennas have better radiation features. In this paper, EcAA is used to study the design issues which are constructed from a combination of linear and elliptical antenna arrays. The overall antenna array structure is displayed in Fig. 1, and the radiation characteristics of an antenna are given by the array factor (AF). Hence, the overall radiation pattern of the EcAA is the multiplication of the linear array factor and elliptical array factor described in $[3,17,21]$.

In this paper, three ellipses are taken with a combination of 12 linear arrays. The ellipses do have equal semi-major axis and semi-minor axis a and b, respectively. The elements along the vertical line form a linear array, and the elements along the transversal plane create an elliptical array.

In general, attractive antenna performance is achieved by turning some of the array elements off while radiating the rest of the elements (Fig. 1). The challenge is to find the exact elements that should be turned on while the remaining turned off. With the help of the proposed optimization technique, the exact state and position of the thinned elements are determined.

\section{Beamforming}

Beamforming creates signals at particular angles to experience constructive interference while others experience destructive interference. Hyper beamforming is a signal processing technique used to control the directionality of the transmission and reception of the radio signals. This greatly improves the gain of the wireless link which increases the range, rate, and penetration capability of the signal. Hence, for deeper SLL reduction

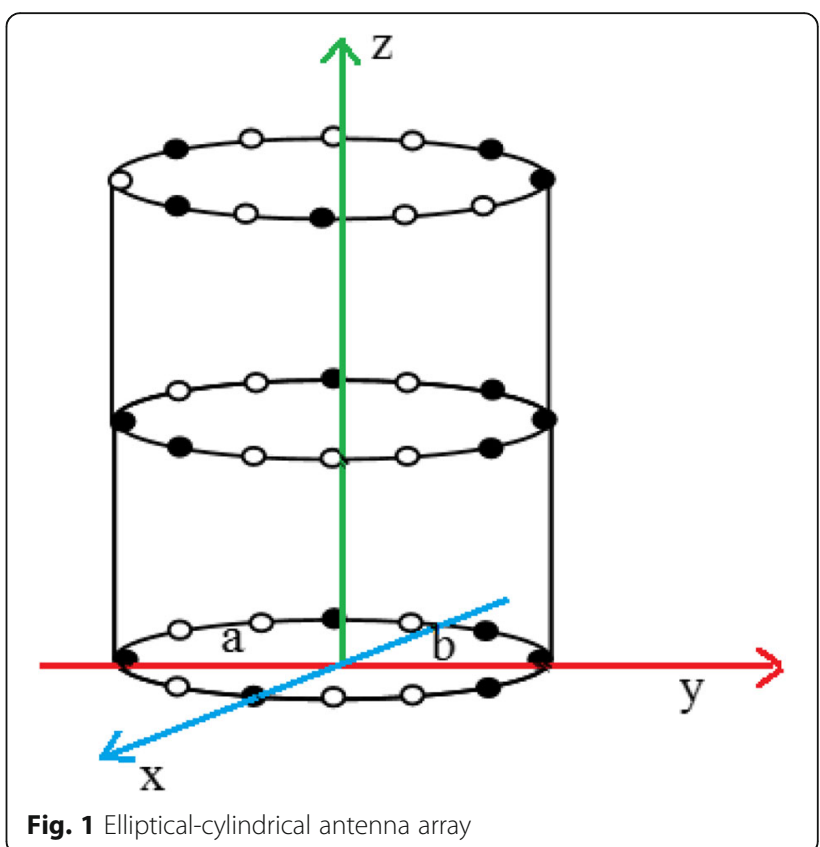


and mulling to the interference direction, hyper beamforming is applied.

The hyper beam is formed by the difference and sum of the radiation pattern of the two half beams which are given in (1) and (2), respectively:

$$
\operatorname{DBeam}(\theta, \phi)=\mid \text { LeftBeam-RightBeam } \mid
$$

$$
\text { SumBeam }(\theta, \phi)=\mid \text { LeftBeam }|+| \text { RightBeam } \mid
$$

Now, the total hyper beam equation is formed by summing the DBeam and SumBeam of the hyper beams and it is a function of the hyper beam exponent, $k$. The total hyper beam equation is given in (3):

$$
\begin{array}{r}
\text { TotalBeam }=\left\{(\mid \text { LeftBeam }|+| \text { RightBeam } \mid)^{k}\right. \\
\left.-(\mid \text { LeftBeam-RightBeam } \mid)^{k}\right\}^{\frac{1}{k}}
\end{array}
$$

The sum and difference patterns of the left beam and right beam are derived from the array factor of the proposed antenna array, given in (4):

$$
\operatorname{AF}_{\text {Total }}(\theta, \phi)=\sum_{m=1}^{M} \sum_{n=1}^{N} C_{m n} \mathrm{e}^{j\left(w \times \sin \theta\left(a \cos \phi \times \cos \phi_{n}+b \sin \phi \sin \phi_{n}\right)\right)}
$$

where DBeam is the difference of the left beam (LeftBeam) and right beam (RightBeam). The SumBeam is the sum of the LeftBeam and RightBeam. The array factor (AF) is evaluated over the elevation and azimuthal angles, and $\phi$, respectively. The $C_{m n}$ is the excitation amplitudes of the array elements where the subscript $m$ stands for the linear elements and $n$ stands for the elliptical elements. $M$ and $N$ are the maximum number of the linear and elliptical elements, respectively. In Eq. (4), $a$ and $b$ are the major and minor axes of the ellipse, respectively, and $w$ is the wavenumber of the radiation characteristics.

\section{System model}

Antenna side lobe introduces interference, and it introduces power wastage as part of the power can be directed to an unintended direction. One solution of this problem is to reduce the number of turned on elements of the array. The optimal number of turned on elements increases the gain and directivity of the antenna. Besides, this minimizes the power wastage and at the same time it reduces the SLL of the antenna system. In both cases, the interference can be decreased and the penetration capability of the signal can be increased. The main inputs of this paper are described in phases 1 and 2 (labeled as A and B in the following subsections):

\section{A. Optimization algorithm description}

PSO works in the manner of bird flocking. All of the particles have fitness values which are evaluated by the fitness function to be optimized, and have velocities which direct the flying of the particles. PSO is initialized with a group of random particles and searches for an optimal solution by updating generations. In every iteration, each particle is updated using two "best" values: the best solution it has achieved so far and the best solution obtained so far by any particle in the whole set.

The pseudocode of the PSO is:

1. For each particle

2. Initialize particle

3. End

4. While maximum iteration is not attained

5. Do

6. For each particle

7. Calculate fitness

8. If the fitness value is greater than the previous private best

9. Transfer the current value as the new private best

10. End

11. Choose the particle with the global best fitness

12. For each particle

13. Update velocity particle

14. Update position particle

15. End

Self-adaptive differential evolution $(\mathrm{SaDE})$ is another intelligent learning algorithm that does not depend on the strategies and parameter settings; rather, the parameters and strategies are made to adapt themselves. Finding the most suitable strategy and computing its control parameters are bulky. Hence, the SaDE algorithm automatically adapts the learning strategy along with the parameter's settings. A similar system model is discussed in [21] and [27] with the main differences are highlighted:

- Initiation: this basically sets up the minimum and maximum values. These values are used in the starting and ending of the iterations. The evolution continues stage-by-stage in many generations.

- Evaluate population: this persistently selects sample space from the whole population for evaluating if it can satisfy the required SLL reduction

- Trail vector: this is a sample of a particular row and columns to be evaluated if it could really result in a good SLL reduction. 
B. Side lobe attenuation

The side lobe attenuation is an iterative algorithm that is introduced for further reduction of the unwanted minor beams. This technique of decreasing side lobes leads to a more programmable radiation pattern of an antenna array. In this case, only the side lobe samples of the array factor that exceed a side lobe threshold is continuously attenuated while measuring if the required SLL is achieved dynamically. This algorithm accomplishes the side lobe reduction without increasing the first null beam width (FNBW). This can be applied either to an optimized antenna array or to non-optimized array patterns. In both cases, it further reduces the existing side lobe. A simplified skeleton of this technique of SLL attenuation is given in Fig. 2.

In the selective SLL attenuation, the attenuation factors of $\alpha$, with the values of greater than 0 and less than 1 , are used. To keep good precision, an attenuation factor of 0.9 is considered in this study.

\section{Results and discussion}

5.1 The effect of thinning and hyper beamforming

For economical and easier antenna synthesis, turning some of the array elements completely off is applied to enhance the radiation characteristics of the antenna design. Using this thinning of the array elements, the SLL is decreased. This happens because the intercoupling interference among the array elements is decreased. The SaDE-optimized hyper beam keeps the number of actively turned on elements optimal while simultaneously reducing the SLL and increasing the directivity. A plot of the normalized array factor (AF) against the elevational angle for different values of the hyper beam exponent is simulated in Fig. 3.

As it is shown in Fig 3, the thinning of the array elements results in better radiation characteristics than the uniformly excited antenna array. Furthermore, applying the hyper beamforming with an exponent of 0.3 , better SLL reduction, and directivity are achieved. Still, by

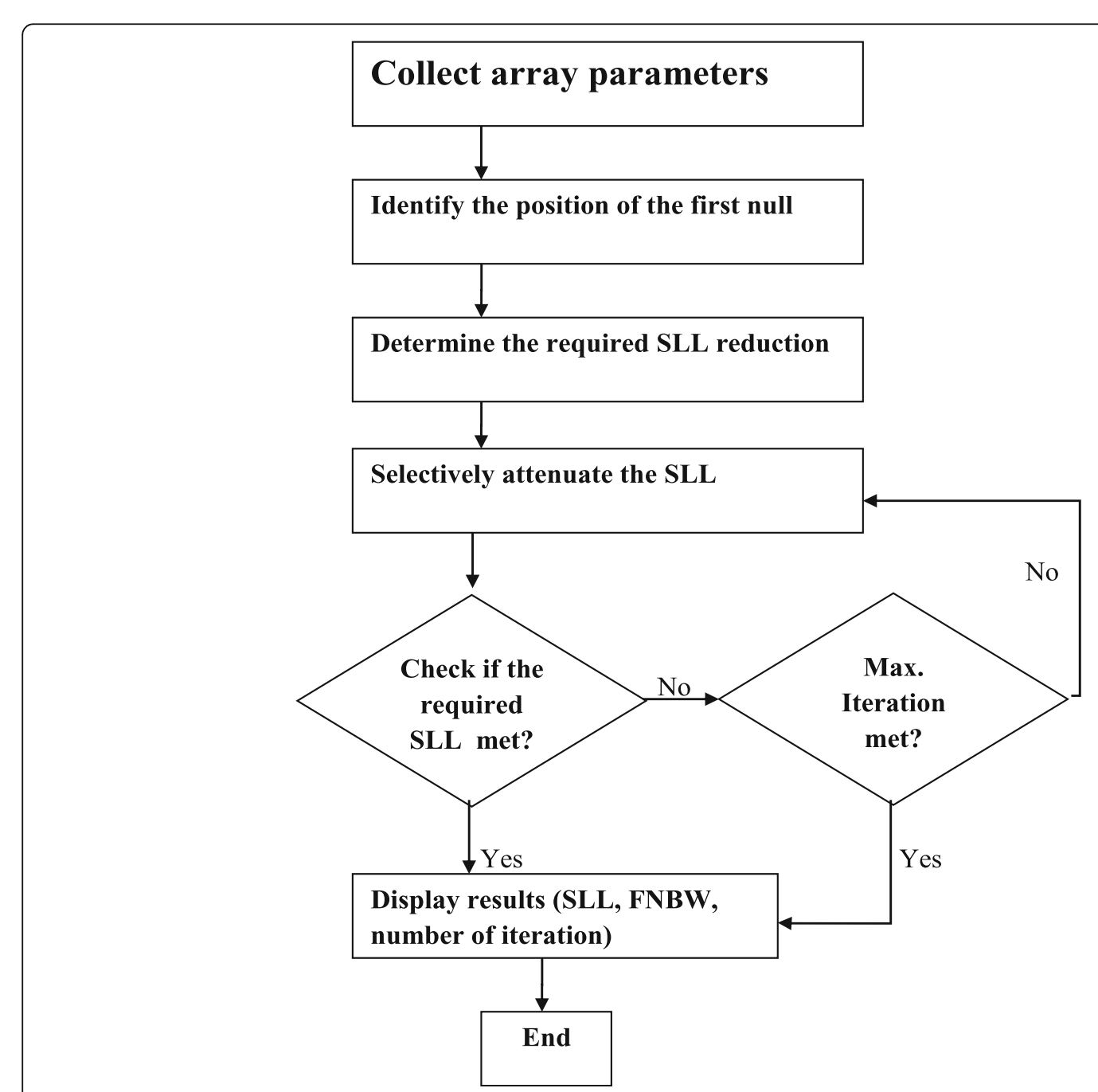

Fig. 2 Simplified SLL attenuation algorithm 


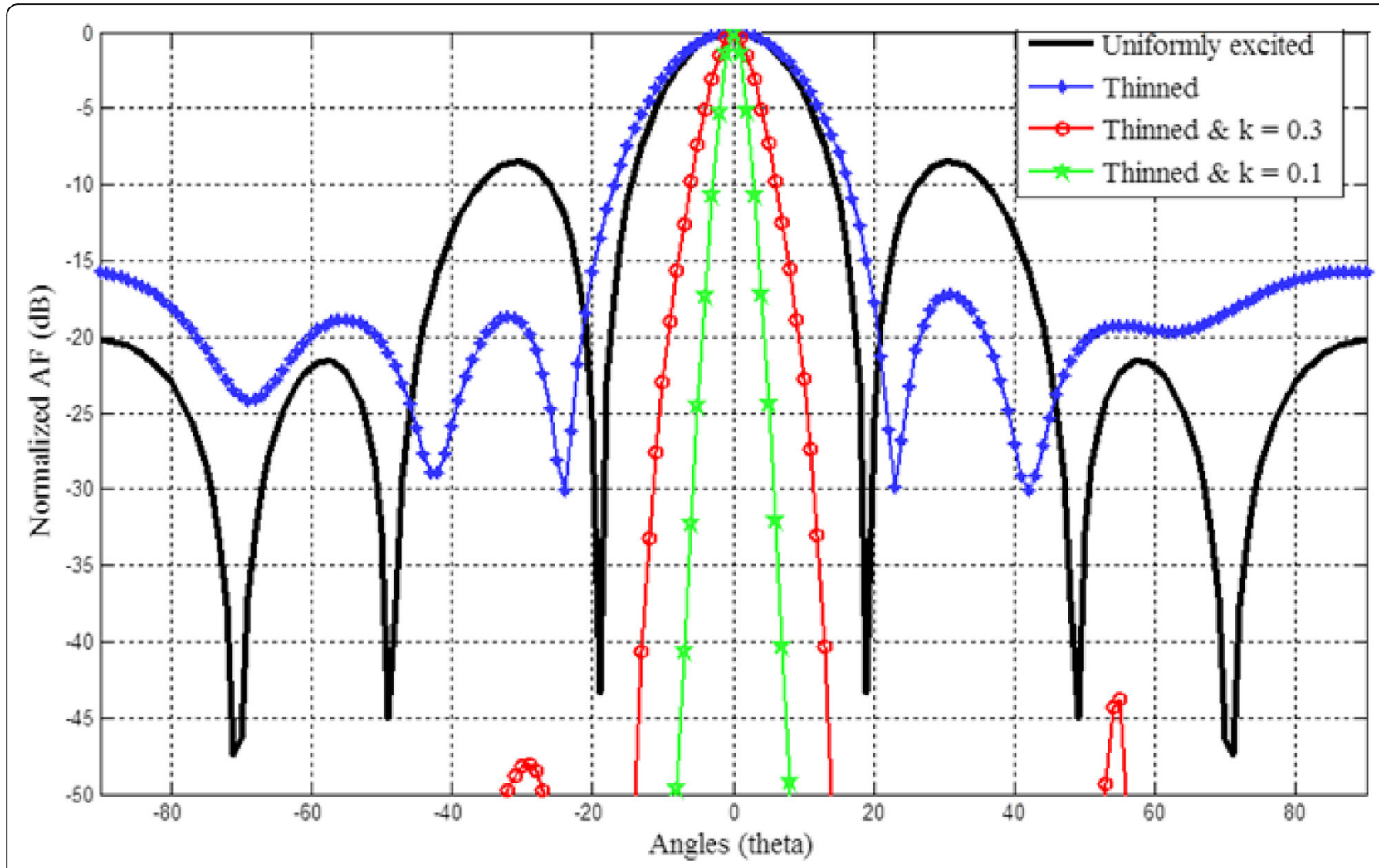

Fig. 3 Thirty-six elements of EcAA with 17 elements turned on

decreasing the hyper beam exponent to 0.1 , a better SLL reduction and increased directivity are simultaneously achieved. The variation of the hyper beam exponent achieves radiation pattern flexibility.

In Fig. 4, the effect of hyper beam exponent on optimized and non-optimized antenna arrays is studied. This SLL reduction is studied on the 15 elements turned on, and we can infer from the following: (i) the optimized plot $(-16.6 \mathrm{~dB})$ indicates that better SLL reduction is possible over the uniform excitation current (ordinary radiation, SLL of $-8.50 \mathrm{~dB}$ ). (ii) Applying a hyper beam with an exponent of 0.2 and 0.1 to both uniform array elements and the thinned array (optimized), the optimized hyper beams have resulted in to a better SLL reduction than the uniform excitation with the same hyper beam exponent.

Similar to the 17 elements turned on (shown in Fig. 3), Fig. 4 illustrates deeper SLL reductions together with a flexible radiation pattern realized when only 15 out of 36 elements are turned on as the hyper beam exponent varies. Comparing Fig. 3 and Fig. 4, the former has more SLL reduction though the latter has a reduced system cost (because a large number of turned on elements result in to more power wastage). Therefore, further increase in the number of turned off elements reduces the radiation intensity of the total array which is not desirable. Hence, the choice of the optimal number of active elements is a trade-off between radiation intensity and interference.

\subsection{The effect of SLL attenuation on EcAA design}

In this paper, we have applied a SLL decreasing mechanism in addition to the optimization and the hyper beamforming techniques. This SLL attenuation-based iterative algorithm attenuates the side lobes without affecting the beam width. In addition to the programmable radiation pattern, this provides substantial benefits by removing unwanted beams of the antenna. The simulation results have depicted that this technique increases the radiation characteristics (reduction of SLL and FNBW) of the antenna significantly. An SLL reduction from -8.50 to $-30 \mathrm{~dB}$ is a result from uniform excitation current as it is shown in Fig. 5, where Imn is the weight of the array elements.

In Fig. 5, it is clear that the SLL is reduced from -32 to $-47 \mathrm{~dB}$. This SLL reduction is evaluated at optimized hyper beam of $k=0.5$ (with only 17 out of the 36 excitation currents of the array elements are turned ON. This indicates that the SLL reduction is further improved when the weighted SLL attenuating mechanism is applied to the optimized array antenna array. This concludes the side lobe minimization mechanism results in to a good SLL reduction without affecting the main beam and hence the combined technique achieves attractive antenna design. 

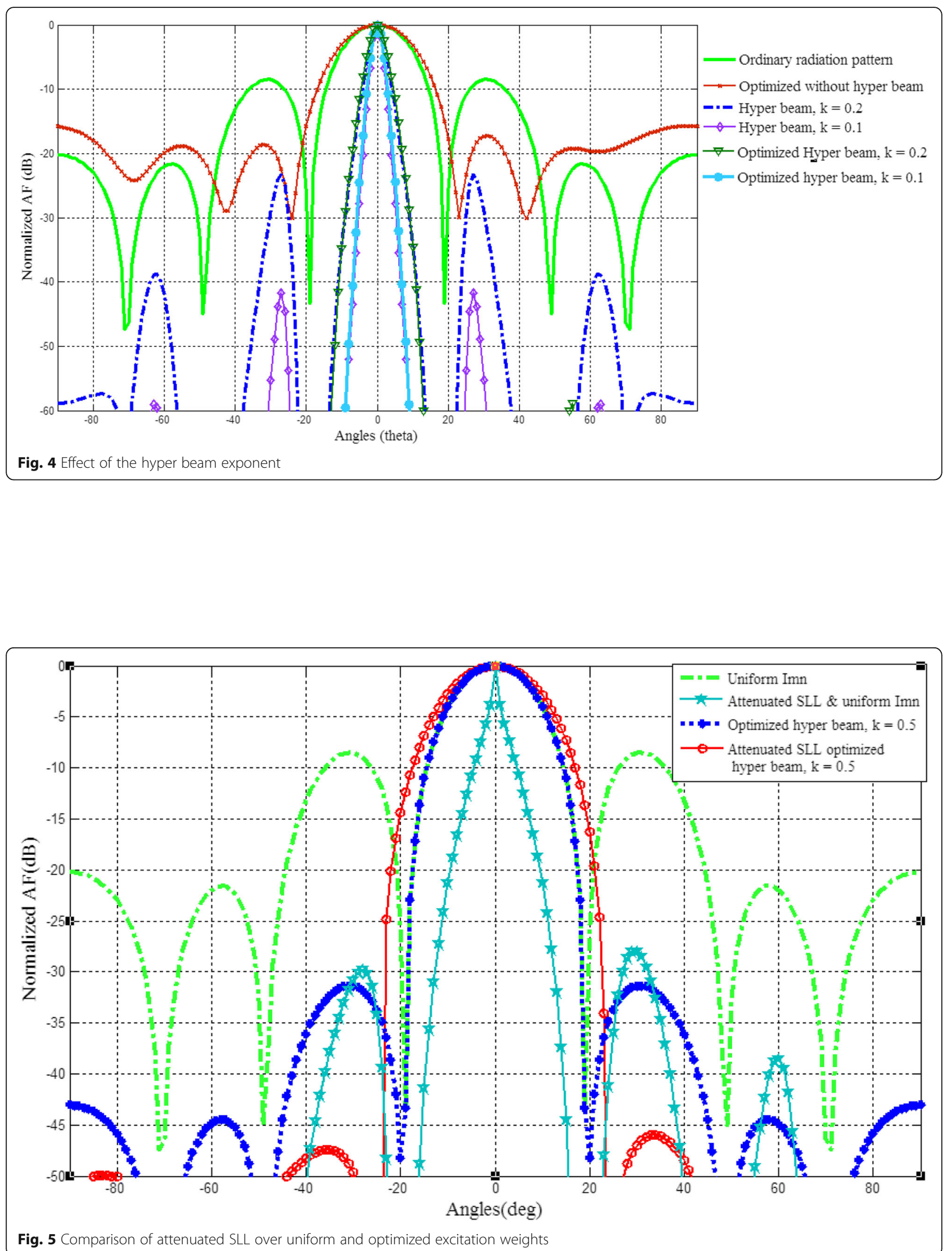

Fig. 5 Comparison of attenuated SLL over uniform and optimized excitation weights 
5.3 Performance comparisons this study with [17]

A similar study has been done for reducing the SLL and power consumption minimization in Bera et al. [17] using PSO as an optimization algorithm. The comparison of this work is done with the results of Bera et al. [17] that are done for SLL reduction on the same antenna array and summarized in Table 1. In Table 1, the experiment number (Exp. No.) 1 is accomplished by particle swarm optimization (PSO) [17] whereas Exp. No. 2, 3, 4, and 5 are done in this paper. The optimized along with the SLL attenuated technique (shown in Exp. No. 5) results in to much better SLL reduction compared to the same hyper beam exponent (shown in Exp. No. 4). This concludes that including the proposed technique of antenna synthesis is worthy enough for better SLL reduction.

In general, from Table 1, the following inferences can be made: (i) in Exp. 2 and 3, the same percent of power conservation is verified as Exp. No. 1 (considering only the number of turned off array elements); however, better SLL reduction is achieved in this work. This demonstrates that the proposed optimization technique and beamforming results in much better SLL reduction than the work done in [17]. (ii) Furthermore, the FNBW is decreased from 74.12 to $36^{\circ}$ and this indicates that the directivity of the proposed antenna is increased. (iii) The power consumption is reduced in this paper as the SLL reduction and increasing directivity reduces the power wastage. (iv) Including the iterative SLL attenuation mechanism further enhances the radiation characteristics of the antenna.

Hence, in this paper, much better radiation characteristics are achieved over [17]. On the other hand, in this paper, further $42.32 \mathrm{~dB}(182.12-139.8 \mathrm{~dB})$ SLL reduction is achieved over the non-uniform excitation antenna [21] at hyper beam exponent of $0.1(k=0.1)$. Moreover, this paper contributes a better practical antenna design than using non-uniform excitation weights of the array elements: (1) unlike the previous work [21], a large number of the array elements can be completely turned off which indicates that a huge amount of power can be conserved; (2) the computation complexity of the thinning of the array elements (in this paper) is simpler whereas the non-uniform excitation takes longer time

Table 1 Comparison of SLL and beam width

\begin{tabular}{lllll}
\hline Exp. no. & EcAA synthesis technique & $\begin{array}{l}\text { Number of } \\
\text { ON elements }\end{array}$ & $\begin{array}{l}\text { SLL } \\
(\mathrm{dB})\end{array}$ & $\begin{array}{l}\text { FNBW } \\
(\mathrm{deg})\end{array}$ \\
\hline 1 & Bera et al. by PSO [17] & 17 & -31.72 & 74.12 \\
2 & Optimized hyper beam, $k=0.3$ & 17 & -44.43 & 38 \\
3 & Optimized hyper beam, $k=0.1$ & 17 & - & 36 \\
& & & 182.12 & \\
4 & Optimized hyper beam, $k=0.5$ & 17 & -30.00 & 36 \\
5 & Attenuated SLL of optimized, & 17 & -47.62 & 36 \\
& $k=0.5$ & & & \\
\hline
\end{tabular}

and hence late for faster design-requiring applications; (3) the deployment of non-uniform excitation antenna design is complex. Therefore, this paper has better simplicity and less power consumption which makes it ideal for antenna design. (4) The concept of SLL attenuation by far achieves better radiation characteristics.

The main concern of [21] is how the proposed algorithm outperforms the rest of the algorithms (GA, PSO, $\mathrm{BBO}$, and IWO) in non-uniformly excited array elements of the antenna. On the other hand, Gebrekrstos et al. [27] discuss the performance of SaDE-optimized hyper beamforming on uniformly excited and thinned antenna arrays. It also evaluates how the SaDE-optimized hyper beamforming performs better than GA. However, in this paper, the main input focuses on how the attenuated SLL outperforms over the uniform and over the thinned excitation weights of the antenna array alone. This work does not discuss the non-uniformly excited array elements of the antenna array. This paper is not mainly concerned with the comparison of different optimization techniques; rather, it confers that the SLL attenuation technique results in much better antenna performance. Generally, this work is closer to [17] which discusses the thinning of the same antenna while the optimization techniques of this paper and [17] are different. As it is seen in Table 1, the radiation characteristics of this paper are significantly better than those of [17]. More specifically, by attenuating the SLL while thinning (turning some of the array elements completely off as determined by the optimization algorithm) along with the beamforming, the excitation weights of the individual array elements are optimized to obtain enhanced antenna performances including reduced SLL, high directivity, reduced power consumption, and a flexible radiation pattern. Hence, this paper has significant contribution to the scientific community as the side lobe attenuation mechanism further reduces the SLL of the antenna array in addition to the beamforming and optimization algorithm.

\section{Conclusion}

An optimization technique that has a small number of control parameters together with a hyper beam is integrated to design an elliptical-cylindrical antenna array. The optimized EcAA has resulted in much better SLL reduction than the uniform excitation amplitudes. In addition to the SLL reduction, the proposed technique of pattern synthesis has contributed to increased directivity and reduced power wastage due to the SLL and turned on array element reduction. Conversely, the reduction of unwanted SLL reduces the interference and hence maximizes the signal to noise ratio. Generally, the proposed technique results in to better SLL reduction and increased directivity (also decreases interference) and maximizes the signal to noise ratio, simultaneously. 


\section{Abbreviations}

AF: Array factor; dB: Decibels; EcAA: Elliptical-cylindrical antenna array; FNBW: First null beam width; SaDE: Self-adaptive differential evolution; SLL: Side lobe level

\section{Acknowledgements}

Not applicable

\section{Authors' contributions}

GGL has coordinated, summarized, and carried out the sequence alignment. $\mathrm{DHH}$ has drafted the manuscript. GGL has also conceived and designed the study, and TB has performed the statistical analysis. All authors read and approved the final manuscript

\section{Funding}

The research was not funded.

\section{Availability of data and materials}

We declare that the MATLAB code used for the simulation will not be shared, and we assure that we will send it on demand.

\section{Consent for publication}

We agree to the publication of the paper.

\section{Competing interests}

The authors declare that they have no competing interests.

Received: 19 February 2019 Accepted: 29 August 2019

Published online: 05 September 2019

\section{References}

1. Björnson et al., Massive MIMO: Ten myths and one critical question. IEEE Communications Magazine 54(2), 114-123 (2016)

2. C.A. Balanis, Antenna Theory and Design, 3rd edn. (John Wiley \& Sons, 2005)

3. Neyestanak, AA Lotfi, et al. "Investigation of hybrid elliptical antenna arrays." IET microwaves, antennas \& propagation 2.1 (2008): 28-34.

4. K.R. Mahmoud et al., A comparison between circular and hexagonal array geometries for smart antenna systems using particle swarm algorithm. Progress in Electromagnetics Research, PIER 72, 75-90 (2007)

5. K.L. Du, Pattern analysis of uniform circular array. IEEE Trans. Antennas Propagat. 52(4), 1125-1129 (Apr. 2004)

6. A. D. Munger, et.al "Conical array studies," IEEE Trans. on Antennas and Propagation, vol. 22, no. 1, pp. 35-43, Jan. 1974.

7. T.E. Morton et al., in Proc. of the 36th Southeastern Symposium on System Theory. Pattern synthesis and performance of conical arrays (Sep. 2004), pp. 145-149

8. E. Yaacoub, et.al , "Hybrid linear and circular antenna arrays," Iranian J. of Electrical and Computer Eng., vol. 6, no. 1, pp. 48-54, Winter/Spring 2006.

9. R.J. Mailloux, Phased array architecture for millimeter wave active arrays. IEEE Antennas and Propagation Society Newsletter 28(1), 4-7 (1986)

10. H.E. Schrank, Low sidelobe phased array antennas. IEEE Antennas and Propagation Society Newsletter 25(2), 4-9 (1983)

11. S.P. ApplebaumandD, J. Chapman, Adaptive arrays with main beam constraints. IEEE Transactions on Antennas and Propagation 24(5), 650-662 (1976)

12. Z. Cui, Z. Shi, J. Zeng, in Proceedings of the 3rd International Conference on Swarm, Evolutionary, and MemeticComputing (SEMCCO'10), vol. 6466of LectureNotes in Computer Science. Using social emotional optimization algorithm to direct orbits of chaotic systems (Springer, Chennai, India, 2010), pp. 389-395

13. D. Mandal, et.al, "Wide null control of linear antenna arrays using particle swarm optimization," in Proceedings of the Annual IEEE India Conference: Green Energy, Computing and Communication (INDICON '10), pp. 1-4, Kolkata, India, December 2010.

14. Z. F. Hao, et.al, "A particle swarm optimization algorithm with differential evolution," in Proceedings of the 6th International Conference on Machine Learning and Cybernetics (ICMLC '07), vol. 2, pp. 1031-1035, August 2007.

15. B. Luitel and G. K. Venayagamoorthy, "Differential evolution particle swarm optimization for digital filter design," in Proceedings of IEEE Congress on Evolutionary Computation (CEC '08), pp. 3954-3961, June 2008.

16. J.J. Liang, A.K. Qin, P.N. Suganthan, S. Baskar, Comprehensive learning particle swarm optimizer for global optimization of multimodal functions. IEEE Transactions on Evolutionary Computation 10(3), 281-295 (2006)
17. Rajesh Bera, et.al "Thinned Elliptical Cylindrical Antenna Array Synthesis Using Particle Swarm Optimization" World Academy of, Engineering and Technology International Journal of Electrical Science, Robotics, Electronics and Communications Engineering Vol:8 No:1, 2014.

18. Pappula, Lakshman, and Debalina Ghosh. "Linear antenna array synthesis using cat swarm optimization." AEU-International Journal of Electronics and Communications 68.6 (2014): 540-549.

19. KV Price, et al, "Differential Evolution: A Practical Approach to Global Optimization " 2005

20. Bauernfeind, Thomas, et al. "PEEC-based multi-objective synthesis of nonuniformly spaced linear antenna arrays." IEEE Transactions on Magnetics (2017).

21. Lema, Gebrehiwet Gebrekrstos, Gebremichael T. Tesfamariam, and Mohammed Ismail Mohammed. "A Novel Elliptical-Cylindrical Antenna Array for Radar Applications." IEEE Transactions on Antennas and Propagation64.5 (2016): 1681-1688.

22. M. Panduro, A. Mendez, R. Dominguez, and G. Romero, "Design of nonuniform circular antenna arrays for side lobe reduction using the method of genetic algorithms," Int. J. Electron. Commun. (AEU), Vol. 60, pp. 713-717, 2006

23. Bian, Li, et.al. "Large thinned array design based on multi-objective cross entropy algorithm." Journal of Shanghai Jiaotong University (Science) 20.4 (2015): 437-442

24. U. Singh, R. Salgotra, Pattern Synthesis of Linear Antenna Arrays Using Enhanced Flower Pollination Algorithm. International Journal of Antennas and Propagation 2017 (2017)

25. W.P.M.N. Keizer, "Fast low sidelobe synthesis for large planar array antennas utilizing successive fast Fourier transforms of the array factor," IEEE Trans. Antennas Propagat., vol. 55, no. 3, pp. 715-722, March 2007.

26. W.P.M.N. Keizer, "Element failure correction for a large monopulse phased array antenna with active amplitude weighting," IEEE Trans. Antennas Propagat., vol. 55, no. 8, pp. 2211-2218, August 2007.

27. G. Gebrekrstos, G. T. Tesfamariam, M. Ismail, "Performance evaluation of elliptical-cylindrical antenna array (EcAA) using SaDE optimized hyper beam," Indonesian Journal of Electrical Engineering and Computer Science, vol. 7, no. 1, pp. 178 188, July 2017.

28. Guariglia, Emanuel. "Entropy and fractal antennas." Entropy18.3 (2016): 84.

29. E. Guariglia, Harmonic sierpinski gasket and applications. Entropy 20(9), 714 (2018)

30. G.G. Lema, D.H. Hailu, Feasibility study of antenna synthesis using hyper beamforming. Heliyon 5(2), e01230 (2019)

\section{Publisher's Note}

Springer Nature remains neutral with regard to jurisdictional claims in published maps and institutional affiliations.

\section{Submit your manuscript to a SpringerOpen ${ }^{\bullet}$ journal and benefit from:}

- Convenient online submission

- Rigorous peer review

- Open access: articles freely available online

- High visibility within the field

- Retaining the copyright to your article

Submit your next manuscript at $>$ springeropen.com 